\title{
Water cooling of shocks in protostellar outflows
}

\section{Herschel-PACS map of L1157 ${ }^{\star}$}

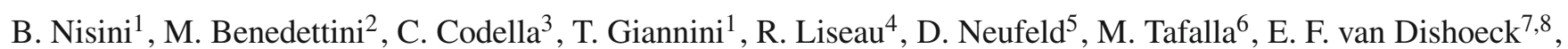
R. Bachiller ${ }^{6}$, A. Baudry ${ }^{9}$, A. O. Benz ${ }^{10}$, E. Bergin ${ }^{11}$, P. Bjerkeli ${ }^{4}$, G. Blake ${ }^{12}$, S. Bontemps ${ }^{9}$, J. Braine $^{9}$, S. Bruderer $^{10}$, P. Caselli ${ }^{13,3}$, J. Cernicharo ${ }^{14}$, F. Daniel ${ }^{14}$, P. Encrenaz ${ }^{15}$, A. M. di Giorgio ${ }^{2}$, C. Dominik ${ }^{16,17}$, S. Doty ${ }^{18}$, M. Fich ${ }^{19}$, A. Fuente ${ }^{6}$, J. R. Goicoechea ${ }^{14}$, Th. de Graauw ${ }^{20}$, F. Helmich ${ }^{20}$, G. Herczeg ${ }^{8}$, F. Herpin ${ }^{9}$, M. Hogerheijde ${ }^{7}$, T. Jacq ${ }^{9}$, D. Johnstone ${ }^{21,22}$, J. Jørgensen ${ }^{23}$, M. Kaufman ${ }^{24}$, L. Kristensen ${ }^{7}$, B. Larsson ${ }^{25}$, D. Lis ${ }^{12}$, M. Marseille ${ }^{20}$, C. McCoey ${ }^{19}$, G. Melnick ${ }^{26}$, M. Olberg ${ }^{4}$, B. Parise ${ }^{25}$, J. Pearson ${ }^{28}$, R. Plume ${ }^{29}$, C. Risacher ${ }^{20}$, J. Santiago ${ }^{6}$, P. Saraceno ${ }^{2}$, R. Shipman ${ }^{20}$, T. A. van Kempen ${ }^{26}$, R. Visser ${ }^{7}$, S. Viti ${ }^{30,2}$, S. Wampfler ${ }^{10}$, F. Wyrowski ${ }^{27}$, F. van der Tak ${ }^{20,31}$, U. A. Yıldız ${ }^{7}$, B. Delforge ${ }^{32,17}$, J. Desbat ${ }^{9,33}$, W. A. Hatch ${ }^{29}$, I. Péron ${ }^{34,32,17}$, R. Schieder ${ }^{35}$, J. A. Stern ${ }^{29}$, D. Teyssier ${ }^{36}$, and N. Whyborn ${ }^{37}$

(Affiliations are available in the online edition)

Received 31 March 2010 / Accepted 23 April 2010

\section{ABSTRACT}

Context. The far-IR/sub-mm spectral mapping facility provided by the Herschel-PACS and HIFI instruments has made it possible to obtain, for the first time, images of $\mathrm{H}_{2} \mathrm{O}$ emission with a spatial resolution comparable to ground based $\mathrm{mm} / \mathrm{sub}-\mathrm{mm}$ observations.

Aims. In the framework of the Water In Star-forming regions with Herschel (WISH) key program, maps in water lines of several outflows from young stars are being obtained, to study the water production in shocks and its role in the outflow cooling. This paper reports the first results of this program, presenting a PACS map of the $\mathrm{o}-\mathrm{H}_{2} \mathrm{O} 179 \mu \mathrm{m}$ transition obtained toward the young outflow L1157.

Methods. The $179 \mu \mathrm{m}$ map is compared with those of other important shock tracers, and with previous single-pointing ISO, SWAS, and Odin water observations of the same source that allow us to constrain the $\mathrm{H}_{2} \mathrm{O}$ abundance and total cooling.

Results. Strong $\mathrm{H}_{2} \mathrm{O}$ peaks are localized on both shocked emission knots and the central source position. The $\mathrm{H}_{2} \mathrm{O} 179 \mu \mathrm{m}$ emission is spatially correlated with emission from $\mathrm{H}_{2}$ rotational lines, excited in shocks leading to a significant enhancement of the water abundance. Water emission peaks along the outflow also correlate with peaks of other shock-produced molecular species, such as $\mathrm{SiO}$ and $\mathrm{NH}_{3}$. A strong $\mathrm{H}_{2} \mathrm{O}$ peak is also observed at the location of the proto-star, where none of the other molecules have significant emission. The absolute $179 \mu \mathrm{m}$ intensity and its intensity ratio to the $\mathrm{H}_{2} \mathrm{O} 557 \mathrm{GHz}$ line previously observed with Odin/SWAS indicate that the water emission originates in warm compact clumps, spatially unresolved by PACS, having a $\mathrm{H}_{2} \mathrm{O}$ abundance of the order of $10^{-4}$. This testifies that the clumps have been heated for a time long enough to allow the conversion of almost all the available gas-phase oxygen into water. The total $\mathrm{H}_{2} \mathrm{O}$ cooling is $\sim 10^{-1} L_{\odot}$, about $40 \%$ of the cooling due to $\mathrm{H}_{2}$ and $23 \%$ of the total energy released in shocks along the L1157 outflow.

Key words. stars: formation - ISM: jets and outflows - ISM: molecules

\section{Introduction}

Among the main coolants in molecular shocks, water is the tracer most sensitive to physical variations and the temporal evolution of protostellar outflows, thus representing a very powerful probe of their shock conditions and thermal history (e.g., Bergin et al. 1998). Water emission and excitation in shocks were studied extensively for the first time with ISO, the first space facility with spectroscopic capabilities in the mid- and far-IR. ISO surveyed the water emission in a large sample of outflows from young stellar objects (YSOs), providing a global statistical picture of the importance of water in the outflow cooling and of variations in its abundance with shock properties and

* Herschel is an ESA space observatory with science instruments provided by European-led Principal Investigator consortia and with important partecipation from NASA. ages (see e.g., Nisini 2003; van Dishoeck 2004). Following ISO, the SWAS and Odin facilities made it possible to observe the ortho- $\mathrm{H}_{2}$ Ofundamental line at $557 \mathrm{GHz}$, providing important constraints on the water abundance and kinematics in the cold outflow gas components (e.g., Franklin et al. 2008; Bjerkeli et al. 2009). All these facilities, however, had poor spatial resolution (i.e. greater than $80^{\prime \prime}$ ), which did not allow one to locate the origin of the water emission nor study variations in abundances and excitation within individual flows.

In this framework, a sample of YSO outflows will be surveyed in different water lines by the PACS and HIFI instruments onboard the Herschel satellite, as part of the key program WISH (Water In Star-forming-regions with Herschel ${ }^{1}$ ). This paper presents the first results obtained from this survey, consisting of a PACS map of the $\mathrm{H}_{2} \mathrm{O} 2_{12}-1_{01} 179 \mu \mathrm{m}$ line covering the

${ }^{1}$ http://wWw.strw. leidenuniv.nl/WISH/ 
outflow of the protostar L1157-mm, obtained during the Herschel science demonstration phase. The $179 \mu \mathrm{m}$ line is the transition connecting the lower two back-bone levels of ortho$\mathrm{H}_{2} \mathrm{O}$. It is therefore one of the brightest water lines expected in collisionally excited conditions, thus representing an ideal tracer of the water distribution in shocked regions. L1157 is a well known outflow driven by a low mass class 0 object (L1157-mm, $L_{\text {bol }} \sim 8.3 L_{\odot}, D=440$ pc, Froebrich 2005). It is considered to be the prototype of chemically active flows, given the large number of different species detected in its shocked regions (e.g., Bachiller \& Perez-Gutierrez 1997). This paper is presenting the first of several observations planned for this source by the WISH team.

\section{Observations}

Observations were performed on 26 October 2009 with the PACS instrument (Poglitsch et al. 2010) onboard the Herschel Space Observatory (Pilbratt et al. 2010) in line spectroscopic mode, with the grating centred on the $\mathrm{H}_{2} \mathrm{O} 22_{12}-1_{01}$ line at $179.527 \mu \mathrm{m}$. The L1157 outflow region (of about $6^{\prime} \times 2^{\prime}$ ) was covered by 3 individual PACS raster maps, arranged along the outflow axis. Each map consists of $3 \times 3$ PACS frames acquired in steps of $40^{\prime \prime}$. The instrument is a $5 \times 5$ pixel array providing a spatial sampling of $9.4^{\prime \prime} /$ pixel, while the spectral resolution at $179 \mu \mathrm{m}$ is $R \sim 1500$ (i.e., $\sim 210 \mathrm{~km} \mathrm{~s}^{-1}$ ). The data were reduced with HIPE 2.0. Additional IDL routines were developed to construct a final integrated and continuum-subtracted line map. Flux calibrations used calibration files obtained by ground tests that remain very uncertain at the time of paper writing, especially for extended sources. To evaluate the flux uncertainty, we compared with the three measurements performed by the ISO satellite along the outflow (Giannini et al. 2001). To do that, we performed aperture photometry of the line emission in the PACS map within the $80^{\prime \prime}$ ISO circular beam. The ratio of PACS to ISO fluxes ranges between 1.1 and 1.8 at the three positions: we adopt this as the uncertainty in our quantitative analysis. The typical rms noise across the map is of the order of $2 \times 10^{-6} \mathrm{erg} \mathrm{s}^{-1} \mathrm{~cm}^{-2} \mathrm{sr}^{-1}$.

\section{Results and comparison with other tracers}

Figure 1 presents the PACS map of the $179 \mu$ m line emission. In the same figure, the $\mathrm{H}_{2} \mathrm{O}$ map is overlaid with contours of the emission from the $\mathrm{H}_{2}$ 0-0 S(1) (Neufeld et al. 2009), CO 2-1 and $\mathrm{SiO} 3-2$ (Bachiller et al. 2001) transitions. The water map exhibits several emission peaks corresponding to the positions of previously-known shocked knots, labelled as B0-B1-B2 for the south east blue-shifted lobe, and R0-R-R2 for the north west red-shifted lobe, following the nomenclature of Bachiller et al. (2001). These emission knots represent the actual working surfaces of a precessing and pulsed jet and are thus associated with the present location of the active shock regions. With respect to $\mathrm{CO}, \mathrm{H}_{2} \mathrm{O}$ emission appears more localized, having a less prominent diffuse component. About $60 \%$ of the total $179 \mu \mathrm{m}$ flux is found within $30^{\prime \prime}$ apertures centered on the knots. This could be partly related to the line excitation: the $179 \mu \mathrm{m}$ line excitation temperature is $\sim 80 \mathrm{~K}$ above the $\mathrm{o}-\mathrm{H}_{2} \mathrm{O}$ ground state (compared to the $17 \mathrm{~K}$ for $\mathrm{CO} 2-1$ ), and the critical density of its upper level is above $10^{8} \mathrm{~cm}^{-3}$ for $T \lesssim 500 \mathrm{~K}$. It may however also be a consequence of the specific conditions needed to ensure a significant production of water. The $\mathrm{H}_{2} \mathrm{O}$ abundance is indeed significantly higher only in shocks strong enough to release the water ice located on grain mantles by sputtering and grain-grain collisions or to activate the gas-phase reactions that convert the gas-phase oxygen into water. Both these processes become efficient at shock velocities $v_{\mathrm{s}} \gtrsim 15 \mathrm{~km} \mathrm{~s}^{-1}$ (Caselli et al. 1997; Jiménez-Serra et al. 2008; Kaufman \& Neufeld 1996). In this respect, we note that the $\mathrm{H}_{2} \mathrm{O}$ emission peaks correspond rather closely to both the position and the relative intensity of the $\mathrm{H}_{2}$ rotational emission (with the $\mathrm{H}_{2} \mathrm{O} 179 \mu \mathrm{m} / \mathrm{H}_{2} \quad 17 \mu \mathrm{m}$ ratio in the range $\sim(2-3) \times 10^{-2}$ for all the $\mathrm{H}_{2}$ peaks $)$. Peaks of low- $J \mathrm{H}_{2}$ pure rotational lines are associated with warm gas (with $T \sim 300-500 \mathrm{~K}$ ) excited in low velocity non-dissociative shocks that are tracers of regions in which a high $\mathrm{H}_{2} \mathrm{O}$ abundance is expected. Other molecules are known to have strongly enhanced abundances in shocks. One of the most well studied of these molecules is $\mathrm{SiO}$, for which Fig. 1 shows that, like water, its emission is very localized around the shocked knots. A similar behavior is found for other molecules, such as $\mathrm{NH}_{3}$ and $\mathrm{CH}_{3} \mathrm{OH}$ (Bachiller et al. 2001; Tafalla \& Bachiller 1995).

The strongest water peak is located at the position of the B1 knot, which is known to be the most chemically active of the L1157 spots (e.g. Bachiller \& Perez Guitierrez 1997; Benedettini et al. 2007; Codella et al. 2010). This knot at near-IR wavelengths appears as a bow shock with intense $\mathrm{H}_{2} 2.12 \mu \mathrm{m}$ emission (Davis \& Eislöffel 1995) and has a significant $\mathrm{H}_{2}$ column density enhancement (Nisini et al. 2010). Although the spatial resolution of the present observations prevents us from completely resolving the bow shock structure, the observed morphology at the B1/B0 positions suggests that water emission is mainly localized at the bow apex and eastern wing. A similar morphology has been observed for molecules such as $\mathrm{SiO}, \mathrm{NH}_{3}$, and CS (Benedettini et al. 2007; Tafalla \& Bachiller 1997), while other shock produced molecules, such as $\mathrm{CH}_{3} \mathrm{OH}$, noticeably have emission localized on the bow western wing (e.g. Codella et al. 2009). This behavior probably relates to an asymmetry in the excitation conditions along the bow structure, most likely induced by the jet precession or the propagation of shocks in an inhomogeneous medium.

Strong, spatially unresolved, water emission is also detected on-source. This localized emission can originate in different components, including shocks impacting on a dense medium at the jet base, the infalling protostellar envelope, or emission from a UV-heated outflow cavity, as discussed in van Kempen et al. (2010) for the HH46-IRS case. The precise origin of this emission will be investigated by dedicated Herschel observations, but we note here the interesting evidence that no other molecule exhibits significant emission at the central position. In particular, the non-detection of strong emission from molecules such as $\mathrm{CH}_{3} \mathrm{OH}$ indicates that grain ice mantle evaporation in the protostellar envelope is unlikely to be the origin of the on-source $\mathrm{H}_{2} \mathrm{O}$ emission, since the two molecules should desorb at similar temperatures. The non-detection of the $\mathrm{H}_{2} 0-0 \mathrm{~S}(1)$ line at the central position is also remarkable. This may be caused by the heavy extinction close to the central source. Assuming an intrinsic $\mathrm{H}_{2} \mathrm{O} 179 \mu \mathrm{m} / \mathrm{H}_{2} 17 \mu \mathrm{m}$ ratio in the range of that observed along the outflow, we estimate that $A_{v}$ on-source should be $\gtrsim 150$ mag to be able to explain the $\mathrm{H}_{2}$ line non-detection. Alternatively, C-type shocks with very high pre-shock densities $\left(\geq 10^{6} \mathrm{~cm}^{-3}\right)$ and velocities between 20 and $40 \mathrm{~km} \mathrm{~s}^{-1}$ are expected to have a large $\mathrm{H}_{2} \mathrm{O} / \mathrm{H}_{2}$ cooling ratio (Kaufman \& Neufeld 1996).

\section{Water abundance and total cooling}

To constrain the range of water column densities that could produce the observed $179 \mu \mathrm{m}$ emission, we consider the SWAS and Odin observations of the $\mathrm{H}_{2} \mathrm{O} 1_{10}-1_{01} 557 \mathrm{GHz}(538 \mu \mathrm{m})$ line observed in this outflow (Franklin et al. 2008; Bjerkeli et al. 2009). Given the large size of the apertures of these two 


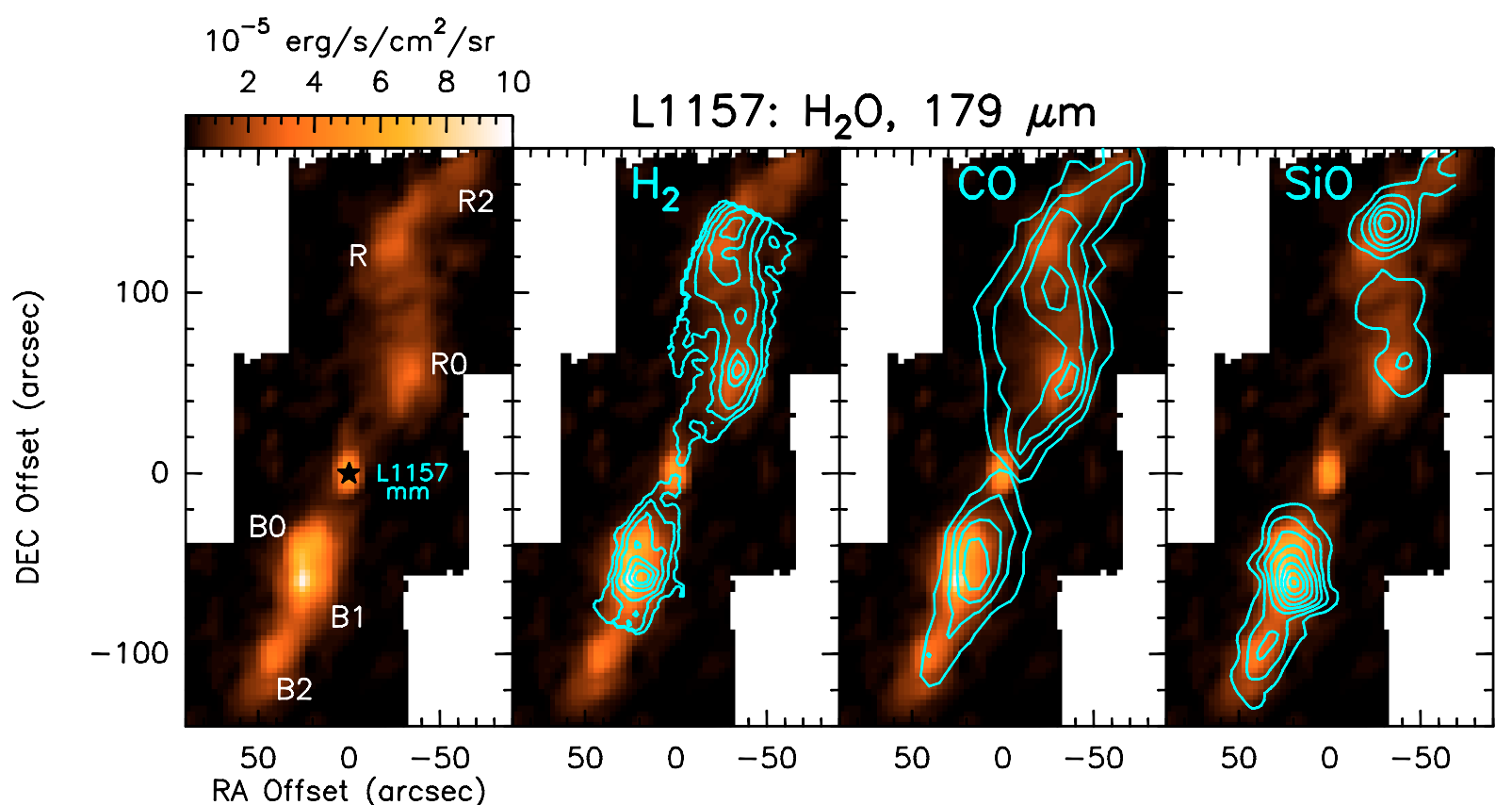

Fig. 1. Continuum subtracted PACS map of the integrated $\mathrm{H}_{2} \mathrm{O} 179 \mu \mathrm{m}$ emission along the L1157 outflow. Offsets are with respect to the L1157-mm source, at coordinates $\alpha(2000)=20: 39: 06.2, \delta(2000)=+68: 02: 16$. The different emission peaks are labelled following the nomenclature adopted by Bachiller et al. (2001) for individual CO peaks. The same map is shown in the other panels with overlays of other tracers, namely $\mathrm{H}_{2}$ 0-0 S(1) at $17 \mu \mathrm{m}$ (Neufeld et al. 2009), CO 2-1, and SiO 3-2 (Bachiller et al. 2001). The spatial resolution of these images are 11" , for $\mathrm{H}_{2}$ and $\mathrm{CO}$, and $18^{\prime \prime}$ for $\mathrm{SiO}$. Note that the $\mathrm{H}_{2}$ observed region does not cover the $\mathrm{B} 2$ and $\mathrm{R} 2$ shocked peaks.

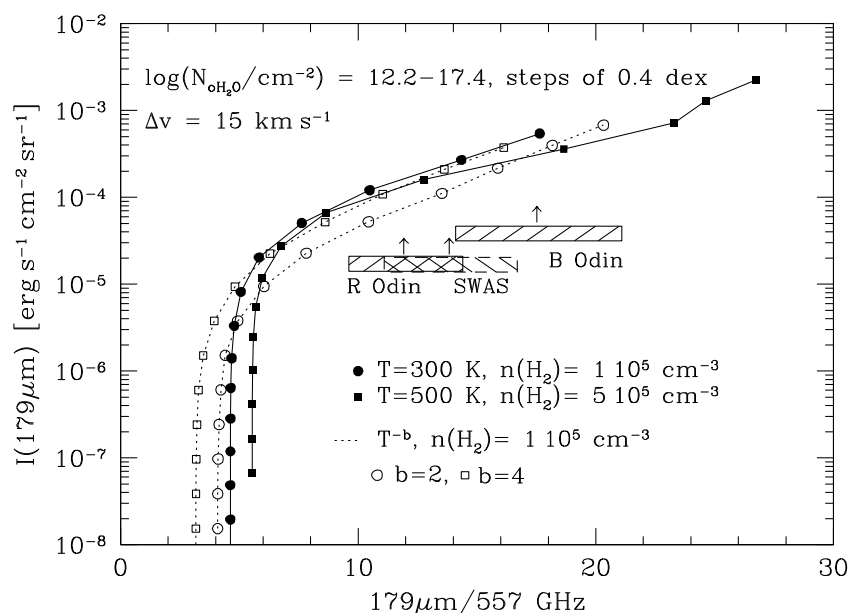

Fig. 2. LVG theoretical predictions of the $179 \mu \mathrm{m}$ line brightness versus the $179 \mu \mathrm{m} / 557 \mathrm{GHz}$ line ratio, compared with observed values. See text for the details.

instruments relative to the PACS spatial resolution, we evaluate here only properties averaged over large outflow regions. In particular, we consider the Odin observations acquired towards the blue (B) and red (R) outflow lobes at offsets $\left(+29^{\prime \prime},-52^{\prime \prime}\right)$ and $\left(-21^{\prime \prime},+121^{\prime \prime}\right)$ (Bjerkeli et al. 2009). The $179 \mu \mathrm{m} / 557 \mathrm{GHz}$ intensity ratios are obtained by diluting the PACS observations to the $126^{\prime \prime}$ Odin resolution. The same procedure was adopted for the SWAS observation that encompasses almost the entire L1157 PACS mapped region with its $3.5^{\prime} \times 5.0^{\prime}$ elliptical aperture. Figure 2 presents large velocity gradient (LVG) predictions, assuming a slab geometry, of the $179 \mu \mathrm{m}$ line brightness versus the $179 \mu \mathrm{m} / 557 \mathrm{GHz}$ line ratio, compared to the observations combined above. The absolute brightnesses are those averaged within an area enclosing $90 \%$ of the total PACS emission inside each considered Odin/SWAS aperture. These emitting areas are $5.9 \times 10^{-8}, 8.0 \times 10^{-8}$, and $2.7 \times 10^{-7}$ sr for the R, B, and the SWAS apertures, respectively. The line intensity derived in this way was considered to be a lower limit to the true $179 \mu \mathrm{m}$ brightness if the PACS emission originates in a clumpy medium, of which the clump size is smaller than the Herschel diffraction limit at $179 \mu \mathrm{m}$.

In the figure, observations are indicated as boxes that take into consideration the uncertainty of a factor of about 1.5 in the $179 \mu \mathrm{m}$ flux, estimated by comparing with the ISO observations (Sect. 2). Theoretical curves were derived as a function of the o$\mathrm{H}_{2} \mathrm{O}$ column density, using the RADEX code (Van der Tak et al. 2007) assuming temperature and density conditions measured from the $\mathrm{H}_{2}$ Spitzer observations or ground-based millimeter observations (Nisini et al. 2010, 2007; Mikami et al. 1992). The temperature is between 300 and $500 \mathrm{~K}$ and the density is in the range $1-5 \times 10^{5} \mathrm{~cm}^{-3}$, the blue lobe being on average colder and denser than the red lobe. Part of the $557 \mathrm{GHz}$ emission can arise from a gas colder than these assumed values, given the lower excitation temperature of this line with respect to the $179 \mu \mathrm{m}$ line. To evaluate the effect of different temperature components along the line of sight on the ratio of the two considered transitions, Fig. 2 also plots the theoretical predictions assuming a temperature stratification where the column density in each layer at a given $T$ varies as $T^{-b}$ (Neufeld \& Yuan 2008). A minimum and maximum temperature of $100 \mathrm{~K}$ and $4000 \mathrm{~K}$, respectively are assumed, and $b$ values between 2 and 4, i.e., the range of values that consistently fit the $\mathrm{H}_{2}$ rotational lines (Neufeld et al. 2009). These curves give the same range of predicted values as the single $T$ curves, indicating that contributions from hightemperature gas do not significantly affect the considered transitions.

Several general conclusions can be drawn from the inspection of Fig. 2. Firstly, the data are consistent with model predictions only if we assume that the real emitting areas are smaller than those estimated from the PACS map. In particular, agreement with the theoretical curves is found for covering factors $\left(f_{\mathrm{c}}\right) \sim 0.1-0.2$, which suggests that the emission is 
Table 1. Estimated water abundances.

\begin{tabular}{ccccccc}
\hline \hline \multirow{2}{*}{$R^{a}$} & $\begin{array}{c}T^{b} \\
\mathrm{~K}\end{array}$ & $\begin{array}{c}n\left(\mathrm{H}_{2}\right)^{b} \\
\mathrm{~cm}^{-3}\end{array}$ & $\begin{array}{c}N\left(\mathrm{oH}_{2} \mathrm{O}\right) \\
10^{16} \mathrm{~cm}^{-2}\end{array}$ & $\begin{array}{c}X\left(\mathrm{H}_{2} \mathrm{O}\right)^{b} \\
10^{-4}\end{array}$ & $\begin{array}{c}A^{c} \\
\mathrm{sr}\end{array}$ \\
\hline $\mathrm{B}$ & $14-20$ & 300 & $3 \times 10^{5}$ & $3-9$ & $0.6-3$ & $\sim 5.2 \times 10^{-9}$ \\
$\mathrm{R}$ & $10-14$ & 500 & $1 \times 10^{5}$ & $2-4$ & $0.8-2$ & $\sim 9.4 \times 10^{-9}$ \\
\hline
\end{tabular}

Notes. ${ }^{(a)} 179 \mu \mathrm{m} / 557 \mathrm{GHz}$ ratio within the Odin aperture. ${ }^{(b)}$ See text for references and assumptions on $T, n$ and $N\left(\mathrm{H}_{2}\right) .{ }^{(c)}$ Effective emission area that reconciles the observed and predicted $179 \mu \mathrm{m}$ line intensity within the Odin aperture.

concentrated on some unresolved emission knots that together do not fill an area larger than a few tens of arcsec. This is not unexpected, since interferometric $\mathrm{mm}$ observations illustrate the extreme clumpiness of the shocked gas, individual knots being of sizes of a few arcsec each (e.g., Benedettini et al. 2007; Lefloch et al. 2010). We note that the typical length scale for planar C-type shocks at the considered densities is of the order of $10^{16} \mathrm{~cm}$, i.e., about $1 / 10$ of the PACS spatial resolution at $D=440 \mathrm{pc}$. The observed $179 \mu \mathrm{m} / 557 \mathrm{GHz}$ ratios, ranging between 10 and 20, are consistent with $N\left(\mathrm{H}_{2} \mathrm{O}\right) \sim 2-9 \times 10^{16} \mathrm{~cm}^{-2}$ (assuming a $\Delta v=15 \mathrm{~km} \mathrm{~s}^{-1}$ from the $557 \mathrm{GHz}$ line width). The $\mathrm{H}_{2}$ column densities, averaged within the PACS emitting areas, were measured from the $\mathrm{H}_{2}$ mid-IR rotational lines and results in $\sim 5 \times 10^{19} \mathrm{~cm}^{-2}$ in both regions covered by the $\mathrm{B}$ and $\mathrm{R}$ observations. The water abundance in the unresolved clumps is therefore estimated to be $\sim N\left(\mathrm{H}_{2} \mathrm{O}\right) / N\left(\mathrm{H}_{2}\right) \times f_{\mathrm{c}} \sim 0.6-3 \times 10^{-4}$ (with $\mathrm{H}_{2} \mathrm{O} \mathrm{o} / \mathrm{p}$ ratio of 3 ). Table 1 reports in more detail the range of values derived in each considered aperture. The total mass of the shocked gas involved in the $179 \mu \mathrm{m}$ emission is of the order of $5 \times 10^{-3} M_{\odot}$, which is only a small fraction $(\sim 1 / 100)$ of the total mass of the outflow estimated from CO observations (e.g. Bachiller et al. 2001). Lefloch et al. (2010) show that $\mathrm{H}_{2} \mathrm{O}$ components with different velocities are discernible in the $557 \mathrm{GHz}$ data acquired by HIFI in a 40" beam centred on the L1157-B1 knot. They separately analyse the different velocity components, confirming that small filling factors are required to explain their observations and finding that the component of higher velocity is the one exhibiting the water abundance of the order of $10^{-4}$. Lower $\mathrm{H}_{2} \mathrm{O}$ abundance values, between $10^{-6}$ and $10^{-5}$, were estimated using only the SWAS and Odin $557 \mathrm{GHz}$ emission, assuming that the $557 \mathrm{GHz}$ emission originates in the same cool gas traced by the low- $J$ $\mathrm{CO}$ emission, thus a gas with a larger covering factor and lower temperature than considered here (Neufeld et al. 2000; Franklin et al. 2008; Bjerkeli et al. 2009). Combining ISO$179 \mu \mathrm{m}$ emission and SWAS observations, Benedettini et al. (2002) derived a water abundance for the warm shocked gas of $\sim 5 \times 10^{-5}$, thus in the lower range of values estimated in the present analysis. However, the ISO observations did not cover the entire L1157 outflow $179 \mu \mathrm{m}$ emission, and the inferred ISO $179 \mu \mathrm{m} / \mathrm{SWAS} 557 \mathrm{GHz}$ ratio was underestimated by about a factor of 2 . Given the considered conditions, the $179 \mu \mathrm{m}$ line contributes to about $30-40 \%$ of the water emission in the outflow: the total estimated $\mathrm{H}_{2} \mathrm{O}$ luminosity is $\sim 8-9 \times 10^{-2} L_{\odot}$, which is about $40 \%$ of the total $\mathrm{H}_{2}$ shock luminosity $\left(0.2 \mathrm{~L}_{\odot}\right.$, Nisini et al. 2010) and about $23 \%$ of the total shock cooling in the L1157 outflow, if we also consider the contributions given by $\mathrm{CO}$ and [OI] derived from ISO observations by Giannini et al. (2001). The high water abundance estimated in the present analysis is consistent with predictions of nondissociative shock models, in which water is mainly produced by endothermic reactions, activated at $T \gtrsim 300 \mathrm{~K}$, where all the available gas-phase oxygen is converted into $\mathrm{H}_{2} \mathrm{O}$, or by the sputtering of icy grain mantles behind the shock. According to Bergin et al. (1998), the time needed to complete this process is of the order of $10^{3} \mathrm{yr}$, for $T=400 \mathrm{~K}$. This is comparable to the shock timescales estimated from $\mathrm{H}_{2}$ observations of individual emission knots of the L1157 outflow (Nisini et al. 2010), thus supporting the idea that the water in this outflow has had time to reach its maximum allowed abundance.

\section{Conclusions}

We have presented a PACS spectral map of the $\mathrm{H}_{2} \mathrm{O} 179 \mu \mathrm{m}$ transition obtained toward the L1157 protostellar outflow. Strong water emission peaks have been found at the location of previously-known shocked spots and correlate well with $\mathrm{H}_{2}$ midIR rotational lines, as well as other important shock tracers, such as $\mathrm{SiO}$ and $\mathrm{NH}_{3}$. The absolute $179 \mu \mathrm{m}$ intensity and the intensity ratios with respect to the previously-observed $557 \mathrm{GHz}$ line, indicate that the water emission originates in warm compact clumps, spatially unresolved by PACS, that have a $\mathrm{H}_{2} \mathrm{O}$ abundance of the order of $10^{-4}$. The total $\mathrm{H}_{2} \mathrm{O}$ cooling has been estimated to be of the order of $8-9 \times 10^{-2} L_{\odot}$, representing about $40 \%$ of the cooling due to $\mathrm{H}_{2}$ and $23 \%$ of the total energy released in shocks along the L1157 outflow.

Additional Herschel PACS/HIFI observations of the L1157 outflow are planned by the WISH program. These will enable us to investigate variations in the water abundance within the outflow and correlate these with kinematical information.

Acknowledgements. This program is made possible thanks to the HIFI guaranteed time and the PACS instrument builders.

\section{References}

Bachiller, R., \& Perez Gutierrez, M. 1997, ApJ, 487, L93

Bachiller, R., Pérez Gutiérrez, M., Kumar, M. S. N., \& Tafalla, M. 2001, A\&A, 372, 899

Benedettini, M., Viti, S., Giannini, T., et al. 2002, A\&A, 395, 657

Benedettini, M., Viti, S., Codella, C., et al. 2007, MNRAS, 381, 1127

Bergin, E. A., Neufeld, D. A., \& Melnick, G. J. 1998, ApJ, 499, 777

Bjerkeli, P., Liseau, R., Olberg, M., et al. 2009, A\&A, 507, 1455

Caselli, P., Hartquist, T. W., \& Havnes, O. 1997, A\&A, 322, 296

Codella, C., Benedettini, M., Beltrán, M. T., et al. 2009, A\&A, 507, L25

Codella, C., et al. 2010, A\&A, 518, L112

Davis, C. J., \& Eislöffel, J. 1995, A\&A, 300, 851

Franklin, J., Snell, R. L., Kaufman, M. J., et al. 2008, ApJ, 674, 1015

Froebrich, D. 2005, ApJS, 156, 169

Giannini, T., Nisini, B., \& Lorenzetti, D. 2001, ApJ, 555, 40

Jiménez-Serra, I., Caselli, P., Martín-Pintado, J., \& Hartquist, T. W. 2008, A\&A, 482,549

Kaufman, M. J., \& Neufeld, D. A. 1996, ApJ, 456, 611

Lefloch, B., et al. 2010, A\&A, 518, L113

Mikami, H., Umemoto, T., Yamamoto, S., \& Saito, S. 1992, ApJ, 392, L87

Neufeld, D. A., \& Yuan, Y. 2008, ApJ, 678, 974

Neufeld, D. A., Snell, R. L., Ashby, M. L. N., et al. 2000, ApJ, 539, L107

Neufeld, D. A., Nisini B., Giannini T., et al. 2009, ApJ, 706, 170, N09

Nisini, B. 2003, Ap\&SS, 287, 207

Nisini, B., Codella, C., Giannini, T., et al. 2007, A\&A, 462, 163

Nisini, B., Giannini, T., Neufeld, D., et al., 2010, ApJ, submitted

Pilbratt, G. L., et al. 2010, A\&A, 518, L1

Poglitsch, A., et al. 2010, A\&A, 518, L2

Tafalla, M., \& Bachiller, R. 1995, ApJ, 443, L37

van der Tak, F. F. S., Black, J. H., Schöier, F. L., Jansen, D. J., \& van Dishoeck, E. F. 2007, A\&A, 468, 627

van Dishoeck, E. F. 2004, ARA\&A, 42, 119

van Kempen, T. A., et al. 2010, A\&A, 518, L128 
1 INAF - Osservatorio Astronomico di Roma, Via di Frascati 33, 00040 Monte Porzio Catone, Italy

e-mail: nisini@oa-roma.inaf.it

2 INAF - Istituto di Fisica dello Spazio Interplanetario, Area di Ricerca di Tor Vergata, via Fosso del Cavaliere 100, 00133 Roma, Italy

3 INAF - Osservatorio Astrofisico di Arcetri, Largo E. Fermi 5, 50125 Firenze, Italy

${ }^{4}$ Department of Radio and Space Science, Chalmers University of Technology, Onsala Space Observatory, 43992 Onsala, Sweden

5 Department of Physics and Astronomy, Johns Hopkins University, 3400 North Charles Street, Baltimore, MD 21218, USA

${ }^{6}$ IGN Observatorio Astronómico Nacional, Apartado 1143, 28800 Alcalá de Henares, Spain

${ }^{7}$ Leiden Observatory, Leiden University, PO Box 9513, 2300 RA Leiden, The Netherlands

${ }^{8}$ Max Planck Institut for Extraterestrische Physik, Garching, Germany

9 Université de Bordeaux, Laboratoire d'Astrophysique de Bordeaux, France; CNRS/INSU, UMR 5804, Floirac, France

10 Institute of Astronomy, ETH Zurich, 8093 Zurich, Switzerland

11 Department of Astronomy, The University of Michigan, 500 Church Street, Ann Arbor, MI 48109-1042, USA

12 California Institute of Technology, Division of Geological and Planetary Sciences, MS 150-21, Pasadena, CA 91125, USA

13 School of Physics and Astronomy, University of Leeds, Leeds LS2 9JT, UK

14 Centro de Astrobiología. Departamento de Astrofísica. CSIC-INTA. Carretera de Ajalvir, Km 4, Torrejón de Ardoz. 28850, Madrid, Spain

15 LERMA and UMR 8112 du CNRS, Observatoire de Paris, 61 Av. de l'Observatoire, 75014 Paris, France

16 Astronomical Institute Anton Pannekoek, University of Amsterdam, Kruislaan 403, 1098 SJ Amsterdam, The Netherlands

17 Department of Astrophysics/IMAPP, Radboud University Nijmegen, PO Box 9010, 6500 GL Nijmegen, The Netherlands

18 Department of Physics and Astronomy, Denison University, Granville, OH, 43023, USA
19 University of Waterloo, Department of Physics and Astronomy, Waterloo, Ontario, Canada

20 SRON Netherlands Institute for Space Research, PO Box 800, 9700 $\mathrm{AV}$, Groningen, The Netherlands

${ }^{21}$ National Research Council Canada, Herzberg Institute of Astrophysics, 5071 West Saanich Road, Victoria, BC V9E 2E7, Canada

22 Department of Physics and Astronomy, University of Victoria, Victoria, BC V8P 1A1, Canada

23 Centre for Star and Planet Formation, Natural History Museum of Denmark, University of Copenhagen, Øster Voldgade 5-7, 1350 Copenhagen, Denmark

24 Department of Physics and Astronomy, San Jose State University, One Washington Square, San Jose, CA 95192, USA

25 Department of Astronomy, Stockholm University, AlbaNova, 106 91 Stockholm, Sweden

${ }^{26}$ Harvard-Smithsonian Center for Astrophysics, 60 Garden Street, MS 42, Cambridge, MA 02138, USA

27 Max-Planck-Institut für Radioastronomie, Auf dem Hügel 69, 53121 Bonn, Germany

28 Jet Propulsion Laboratory, California Institute of Technology, Pasadena, CA 91109, USA

29 Department of Physics and Astronomy, University of Calgary, Calgary, T2N 1N4, AB, Canada

30 Department of Physics and Astronomy, University College London, Gower Street, London WC1E6BT, UK

31 Kapteyn Astronomical Institute, University of Groningen, PO Box 800, 9700 AV, Groningen, The Netherlands

32 Institute Laboratoire d'Etudes du Rayonnement et de la Matire en Astrophysique, UMR 8112 CNRS/INSU, OP, ENS, UPMC, UCP, Paris, France

33 CNRS/INSU, UMR 5804, B.P. 89, 33271 Floirac cedex, France

34 Institute Institut de Radioastronomie Millimetrique, IRAM, 300 rue de la Piscine, 38406 St Martin d'Heres, France

35 KOSMA, I. Physik. Institut, Universität zu Köln, Zülpicher Str. 77, 50937 Köln, Germany

36 European Space Astronomy Centre, ESA, PO Box 78, 28691 Villanueva de la Caada, Madrid, Spain

37 ALMA 